\title{
Effectiveness of palliative care for non-small cell lung cancer (Review)
}

\author{
HUIQIN LI ${ }^{1}$ and JIANING LI ${ }^{2}$ \\ Departments of ${ }^{1}$ Internal Medicine Ward 8 and ${ }^{2}$ Radiation Oncology Ward 5, \\ Shandong Tumor Hospital, Jinan, Shandong 250117, P.R. China
}

Received February 8, 2016; Accepted July 4, 2016

DOI: 10.3892/etm.2016.3621

\begin{abstract}
Lung cancer is the leading cause of cancer mortality worldwide. Despite increases in the survival rate for various types of cancer over the past several decades, lung cancer remains an overwhelmingly lethal disease and the majority of patients succumb to the disease in a short period of time. A number of treatment options are available depending on the stage of lung cancer. The present review focused on palliative care and is associated with stage IIIB and IV of non-small cell lung cancer (NSCLC). Stage IIIB disease is not amenable to curative treatment and for stage IV disease, treatment is palliative in nature, with a focus on increasing survival time, controlling symptoms and improving or maintaining quality of life. Palliative treatment options include chemotherapy, radiotherapy and supportive care. The present review examines the important aspects of palliative therapy with regard to NSCLC.
\end{abstract}

\section{Contents}

1. Introduction

2. Palliative care in lung cancer

3. Palliative chemotherapy vs. supportive care alone

4. Comparison of different palliative chemotherapy regimens

5. Patient perspective on survival benefit of palliative chemotherapy

6. Impact of palliative chemotherapy on QOL and symptom levels

7. Conclusion

\section{Introduction}

Lung cancers are divided into two main histological subtypes: small cell (SCLCs) and non-small cell lung cancers (NSCLCs).

Correspondence to: $\mathrm{Dr}$ Huiqin $\mathrm{Li}$, Department of Internal Medicine Ward 8, Shandong Tumor Hospital, 440 Jiyan Road, Jinan, Shandong 250117, P.R. China

E-mail: lihuiqin727@163.com

Key words: palliative care, non-small cell lung cancer, cancer, supportive care
These two types represent cancers that grow and respond to treatment in very different ways and are studied and treated as separate diseases. SCLCs account for $15-20 \%$ of lung cancers, tend to grow rapidly and are classified as either limited or extensive stage $(1,2)$. NSCLC is a collection of several tumour histologies including adenocarcinoma, squamous-cell and large cell carcinomas (3). NSCLC accounts for 80-85\% of all lung cancers and is characterized by retarded growth and spread compared to SCLC (4). NSCLC is staged using the traditional TNM solid tumour staging system, which is based on tumour size, nodal status and presence or absence of metastases (2). TNM staging is used to group NSCLCs more broadly into four stage categories: I, II, III and IV, the first three of which can be subdivided into A and B subtypes. Stage I cancers are confined to the lung and are no larger than $5 \mathrm{~cm}$ while stage II cancers may have some limited spread beyond the primary tumour and are no larger than $7 \mathrm{~cm}$ (2). Stage IIIA cancers are characterized by greater spread within the lung itself or connected organs (excluding the opposite lung) or lymph nodes on the same side of the chest (2). Stage IIIB cancers are those that have greater spread into connecting organs and/or lymph nodes above the collar bone or on the contralateral side of the body (2). Stage IV consists of disease that has metastasized to the opposite lung, the fluid surrounding the lungs or heart or to other more distant parts of the body, including the brain, liver, and bones (2). Stages III (usually restricted to IIIB) and stage IV are frequently collectively referred to as 'advanced' stage disease. Approximately $34 \%$ of patients are diagnosed with stage I or II, $27 \%$ are diagnosed with stage III, and $39 \%$ are diagnosed with stage IV (5).

\section{Palliative care in lung cancer}

Palliative care can be broadly defined as care aimed to improve the quality of life (QOL) of individuals suffering from life-threatening illnesses (4). The World Health Organization (WHO) describes palliative care as 'an approach that improves the QOL of patients and their families facing the problems associated with life-threatening illness, through the prevention and relief of suffering by means of early identification and impeccable assessment and treatment of pain and other problems, physical, psychosocial and spiritual' (6). Palliative care for NSCLC can be subdivided into two main categories: the first category is supportive (patient-centred) care and the 
second category is tumour-directed therapy. Supportive care, which can include antibiotics, corticosteroids, analgesics, antiemetics, transfusions and psychosocial support (7), is targeted directly at improving the wellbeing of the patient. Tumour-directed therapy also aims to improve patient wellbeing but does so indirectly by targeting the cancer itself using strategies that decrease tumour burden, which in turn, improves patient symptoms and wellbeing. Tumour-directed palliative treatment options for NSCLC include palliative chemotherapy and radiotherapy.

For locally advanced unresectable and metastatic NSCLC (stages IIIB and IV, which are unsuitable for curative treatment options), the standard first-line palliative treatment is palliative chemotherapy with supportive care $(8,9)$. Patients not fit enough to undergo chemotherapy are offered supportive care alone. For patients with specific, localized symptoms care may also include a short course of radiotherapy, which has been shown to alleviate symptoms and improve QOL (10).

\section{Palliative chemotherapy vs. supportive care alone}

Clinical trials have shown a survival advantage of palliative chemotherapy compared to supportive care alone (7). A meta-analysis performed by the Cochrane Collaboration, which included 16 trials and represented over $84 \%$ of the patients from all known randomized trials (as of November 2009) reported an absolute median survival improvement of 1.5 months (from 4.5 to 6 months) resulting in an absolute improvement in the one year survival rate of $9 \%$ (from 20 to $29 \%$; $\mathrm{HR}=0.77$, 95\% $\mathrm{CI}=0.71-0.83, \mathrm{p}<0.0001)$ for chemotherapy compared to supportive care alone (7). In patients fit enough to undergo modern, standard, platinum-based, two-drug (platinum doublet) combination chemotherapy, the reported median survival times ranged from 7.4 to 11.3 months (11).

\section{Comparison of different palliative chemotherapy regimens}

A systematic review of first-line systemic chemotherapy for advanced NSCLC included a discussion of 10 previous systematic reviews (12-14) and concluded that platinum-based doublet chemotherapy (PPDC) is the standard of care for first-line chemotherapy, that platinum-based doublets are superior to nonplatinum-based chemotherapies (15), platinum agents alone or other agents alone (13), and that none of the standard platinum doublets is clearly superior to any other. Standard palliative, PPDC regimens include cisplatin or carboplatin combined with gemcitabine, vinorelbine, paclitaxel or docetaxel (16).

\section{Patient perspective on survival benefit of palliative chemotherapy}

While the survival benefit is clear, the magnitude of that benefit is small (17). However, whether such a small gain is important from the patients' perspective remains to be determined. A study of patients previously treated with cisplatin-based chemotherapy for advanced NSCLC reported a median survival threshold for accepting mildly toxic chemotherapy of 4.5 and 9 months for accepting severely toxic chemotherapy (18). When given the choice between supportive care and chemotherapy, only $22 \%$ of those patients selected chemotherapy for its 3-month survival advantage. By contrast, 68\% accepted chemotherapy if it substantially reduced the symptoms (18). The limited survival gains achieved and the relative importance patients place on quality over quantity of life underscores the need for data on QOL and symptom implications of palliative chemotherapy (8) and supports the contention that QOL and symptomatic status are important endpoints in their own right when assessing palliative chemotherapy.

\section{Impact of palliative chemotherapy on QOL and symptom levels}

Traditionally, the primary endpoints in the clinical randomized controlled trial (RCTs) of palliative chemotherapy for advanced NSCLC have been survival and objective tumour response, while QOL and symptom control have been neglected or relegated to secondary objectives (18). Acceptance of QOL measures as important primary endpoints is on the increase and clinical trials of the past decade often report some QOL measurement. In the RCTs that have addressed QOL, a variety of study methods, chemotherapy regimens and measurement instruments have been employed and the ability of chemotherapy to improve QOL is under debate (19). However, the majority of studies using validated QOL tools have reported some QOL (45-49) and symptom benefit (20) in favour of chemotherapy over supportive care alone, or at minimum, no detrimental effect of chemotherapy compared to supportive care $(21,22)$. However, QOL results are usually confined to a few comments regarding whether there was a significant difference between treatment arms or from baseline and a corresponding P-value without any further detail on absolute changes or proportions of patients who obtained improvement or stabilization of their QOL or symptom levels. There is usually no statistically or clinically significant mean change in QOL from baseline (2553-56). It should be noted that given that the natural trajectory of NSCLC for QOL and that symptoms continually decrease, a treatment that merely maintains current QOL and symptom levels and delays progression is considered successful.

\section{Conclusion}

The abovementioned studies indicate that palliative care has been recommended as the standard of care for patients with advanced NSCLC based on the results of random clinical trials completed over the past several decades. These trials have reported improvement or stabilization in QOL in one half to one third of patients. However, more comprehensive studies are required to investigate the effectiveness of this treatment in the clinic. Additionally, whether the efficacy of palliative care demonstrated in the trials translates into similar levels of effectiveness in routine practice remains to be determined.

\section{References}

1. Petersen I and Warth A: Lung cancer: developments, concepts, and specific aspects of the new WHO classification. J Cancer Res Clin Oncol: Jul 22, 2015 (Epub ahead of print).

2. Sobin L and Wittekind C (eds): TNM Classification of Malignant Tumours. 6th edition. John Wiley \& Sons, Hoboken, NJ, 2002. 
3. Chen Z, Fillmore CM, Hammerman PS, Kim CF and Wong KK Non-small-cell lung cancers: A heterogeneous set of diseases. Nat Rev Cancer 14: 535-546, 2014.

4. Canadian Cancer Statistics 2010. Canadian Cancer Society, Toronto, 2010.

5. Morgensztern D, Ng SH, Gao F, Govindan R: Trends in stage distribution for patients with non-small cell lung cancer: a National Cancer Database survey. J Thorac Oncol 5: 29-33, 2010.

6. World Health Organization: WHO definition of palliative care. http://www.who.int/cancer/palliative/definition/en/. 2010.

7. Non-Small Cell Lung Cancer Collaborative Group: Chemotherapy and supportive care versus supportive care alone for advanced non-small cell lung cancer. Cochrane Database Syst Rev 5 : CD007309, 2010.

8. Plunkett TA, Chrystal KF and Harper PG: Quality of life and the treatment of advanced lung cancer. Clin Lung Cancer 5: 28-32, 2003.

9. Goffin J, Lacchetti C, Ellis PM, Ung YC and Evans WK; Lung Cancer Disease Site Group of Cancer Care Ontario's Program in Evidence-Based Care: First-line systemic chemotherapy in the treatment of advanced non-small cell lung cancer: a systematic review. J Thorac Oncol 5: 260-274, 2010.

10. Okawara G, Mackay JA, Evans WK and Ung YC; Lung Cancer Disease Site Group: A Clinical Practice Guideline. In Management of Unresected Stage Ill Non-Small Cell Lung Cancer. A Quality Initiative of the Program in Evidence-based Care (PEBC), Cancer Care Ontario (CCO). Cancer Care Ontario, Toronto, 2006

11. Ramalingam S and Belani C: Systemic chemotherapy for advanced non-small cell lung cancer: recent advances and future directions. Oncologist 13 (Suppl 1): 5-13, 2008.

12. Le Chevalier T, Scagliotti G, Natale R, Danson S, Rosell R, Stahel R, Thomas P, Rudd RM, Vansteenkiste J, Thatcher N, et al: Efficacy of gemcitabine plus platinum chemotherapy compared with other platinum containing regimens in advanced non-small-cell lung cancer: a meta-analysis of survival outcomes. Lung Cancer 47: 69-80, 2005.

13. Hotta K, Matsuo K, Ueoka H, Kiura K, Tabata M and Tanimoto M: Addition of platinum compounds to a new agent in patients with advanced non-small-cell lung cancer: a literature based metaanalysis of randomised trials. Ann Oncol 15: 1782-1789, 2004.
14. Hotta K, Matsuo K, Ueoka H, Kiura K, Tabata M and Tanimoto M: Meta-analysis of randomized clinical trials comparing cisplatin to carboplatin in patients with advanced non-small-cell lung cancer. J Clin Oncol 22: 3852-3859, 2004.

15. Baggstrom MQ, Stinchcombe TE, Fried DB, Poole C, Hensing TA and Socinski MA: Third-generation chemotherapy agents in the treatment of advanced non-small cell lung cancer: a meta-analysis. J Thorac Oncol 2: 845-853, 2007.

16. Azzoli CG, Baker S Jr, Temin S, Pao W, Aliff T, Brahmer J, Johnson DH, Laskin JL, Masters G, Milton D, et al; American Society of Clinical Oncology: American Society of Clinical Oncology Clinical Practice Guideline update on chemotherapy for stage IV non-small-cell lung cancer. J Clin Oncol 27: 6251-6266, 2009.

17. Temel J: Complexities of quality of life analysis in non-small cell lung cancer. J Support Oncol 5: 30-31, 2007.

18. Silvestri G, Pritchard R and Welch HG: Preferences for chemotherapy in patients with advanced non-small cell lung cancer: descriptive study based on scripted interviews. BMJ 317: 771-775, 1998.

19. Stinnett S, Williams L and Johnson DH: Role of chemotherapy for palliation in the lung cancer patient. J Support Oncol 5: 19-24, 2007.

20. Roszkowski K, Pluzanska A, Krzakowski M, Smith AP, Saigi E, Aasebo U, Parisi A, Pham Tran N, Olivares R and Berille J: A multicenter, randomized, phase III study of docetaxel plus best supportive care versus best supportive care in chemotherapy-naive patients with metastatic or non-resectable localized non-small cell lung cancer (NSCLC). Lung Cancer 27: $145-157,2000$

21. Brown J, Thorpe H, Napp V, Fairlamb DJ, Gower NH, Milroy R, Parmar MK, Rudd RM, Spiro SG, Stephens RJ, et al: Assessment of quality of life in the supportive care setting of the big lung trial in non-small-cell lung cancer. J Clin Oncol 23: 7417-7427, 2005.

22. Tanvetyanon T, Soares HP, Djulbegovic B, Jacobsen PB and Bepler G: A systematic review of quality of life associated with standard chemotherapy regimens for advanced non-small cell lung cancer. J Thorac Oncol 2: 1091-1097, 2007. 